\title{
THE NATURE OF THE GALACTIC CENTER ARC
}

\author{
E. SERABYN \\ Department of Physics, Mathematics and Astronomy \\ California Institute of Technology, Pasadena, CA 91125
}

\begin{abstract}
Ever since the Galactic Center Arc was resolved into its component filaments a decade ago, it has been clear that its linear structure arises from the influence of a strong magnetic field. However, the origin and nature of the contributory phenomena have remained elusive. Since what is seen is synchrotron emission from relativistic particles, of prime interest is a knowledge of the acceleration mechanism involved. Interferometric imaging of the molecular gas in the vicinity of the Arc has now provided a tantalizing clue to the Arc's origin: molecular clumps coinciding with the endpoints of a number of the Arc's filaments point to these clumps as the source of the relativistic particles. This suggests that as dense molecular clumps course through the ambient magnetic field at the Galactic Center, magnetic energy is liberated in their leading layers via field reconnection, precipitating rapid acceleration of free charges to high energy.
\end{abstract}

\section{Introduction}

The resolution of the Galactic Center Arc (the "Arc" in the following) into a set of a dozen or so linear nonthermal filaments (NTFs) has contributed to a gradual, but fundamental change in our view of the Galactic center environment. While the synchrotron nature of the Arc's radio emission demands the presence of both a strong magnetic field and a copious supply of relativistic particles, numerous observations of the central molecular cloud layer have brought additional insights, particularly concerning the turbulent nature of these clouds. As is outlined in the following, an understanding of the nature of the Galactic Center Arc can be achieved from a synthesis of our knowledge in these two areas. I begin with a short review of both topics. A more complete review, including a compendium of extant models, 
is provided by M. Morris in this volume.

The presence of a high strength magnetic field in the central hundred or so parsecs of our Galaxy is observationally well supported (Morris 1994), but the field morphology and strength nevertheless remain subject to considerable uncertainty. Thus, while it is now known that the ambient field is oriented roughly perpendicular (vertical) to the plane of the Galaxy in the NTFs making up the Arc, as wel as in several other 'threads' of nonthermal emission in the vicinity of the Galactic Center (Morris, this volume), it is not yet known if these nonthermal filaments correspond to regions of enhanced field strength (flux tubes), or to sites of particle injection in a more or less uniform vertical field. In terms of field strengths, although estimates for the central region span a large range, from tens of $\mu \mathrm{G}$ to several mG (Yusef-Zadeh et al. 1984; Sofue et al. 1987), all of the measured values are well above typical $\mu \mathrm{G}$ Galactic disk values. Thus, the general picture of a strong, vertically oriented magnetic field in the central region of our Galaxy has become established.

Concurrently, our views of the molecular cloud layer at the Galactic center have evolved, from earlier resolution-limited overviews of the large scale gas distribution and kinematics, to quite detailed views of the physical conditions and kinematics of individual clouds, and of the molecular cloud population as a whole. Thus, it is now clear that molecular clouds in the central few hundred parsecs differ considerably from their counterparts in the large-scale Galactic disk. Physically, the Galactic Center clouds are both hotter and denser (Güsten 1989). Moreover, external forces acting on the clouds are much larger in the Galactic Center: both tidal gravitational forces and magnetic pressures are much higher. Finally, the Galactic Center clouds are invariably more turbulent: they have both higher internal velocity dispersions and also a much higher random component to their systemic velocities, with some clouds following orbits decidedly at odds with systematic rotation (Bally et al. 1989). Indeed, many clouds show radial velocities which are "forbidden", i.e., opposite in sign to Galactic rotation. Thus, the molecular medium in our Galactic nucleus consists of an ensemble of clouds which is much more chaotic, more highly excited, and more affected by large scale external forces than are the clouds in the Galactic disk.

Given a strong vertical magnetic field and a sub-population of anomalously orbiting molecular clouds (i.e., clouds on orbits not in accord with corotation of the interstellar medium and the magnetic field), it is natural to ask how the two interact. In particular, what happens as these anomalously orbiting clouds (which presumably have some fractional ionization level) attempt to traverse the vertical magnetic field lines? This question becomes all the more interesting when it is revealed that an anomalously orbiting molecular cloud is located at the approximate midpoint of the 
Galactic Center Arc. I return to this issue after briefly discussing some of the most relevant observational data relating this cloud to the Arc.

\section{Molecular Observations}

The molecular cloud in question, M0.20-0.033 or the $25 \mathrm{~km} \mathrm{~s}^{-1}$ cloud, lies roughly in the true Galactic plane, near the midpoint of the nonthermal filaments which comprise the Galactic Center Arc (Lasenby et al. 1989; Serabyn \& Güsten 1991 - hereafter SG). Its radial velocity, $25 \mathrm{~km} \mathrm{~s}^{-1}$, is difficult to reconcile with simple Galactic rotation if the Arc is actually as close to the Galactic Center as it appears in projection. Two lines of argument suggest that this cloud is interacting with the Arc. The most obvious mode of interaction is through the mediation of the HII region G0.18-0.04 (Yusef-Zadeh \& Morris 1987), which appears to 'wrap around' the sheaf of NTFs making up the Arc. As this HII region represents the ionized surface of the $25 \mathrm{~km} \mathrm{~s}^{-1}$ cloud (SG), the molecular cloud is obviously also involved in the interaction. Moreover, as molecular material seems to 'protrude' from the main body of the $25 \mathrm{~km} \mathrm{~s}^{-1}$ cloud along the direction of the NTFs in the single dish CS map (SG), a more direct interaction between the molecular medium and the NTFs is also intimated.

While single dish observations thus provided an overview of the molecular cloud morpholgy and suggested the model components to be discussed below (SG), recent observations with the OVRO $\mathrm{mm}$-wave interferometer have provided further clues which have put these hypotheses on a stronger observational footing (Serabyn \& Morris 1994 - hereafter SM). Fig. 1 presents the interferometric image of the emission from the $25 \mathrm{~km} \mathrm{~s}^{-1}$ cloud in the CS $J=2-1$ line. Because of the small primary beam, the mapped fields cover only a small fraction of the cloud, but the three fields available are nevertheless quite revealing.

As discussed in more detail in SM, several molecular clumps are seen in the mapped fields, all of which lie athwart one or more of the nonthermal filaments. Indeed, only a few of the filaments pass by these clumps without crossing one or another of them, and of course even these filaments may encounter a molecular clump outside of the mapped interferometer fields. More importantly however, the observed clumps all lie close to the locations at which the NTFs either fade from view, or change direction slightly. Thus, in brief, it is possible to conclude that the endings and bendings of the NTFs near G0.18-0.04 are intimately related to the presence of molecular gas clumps.

Does this mean that these clumps are sites of relativistic particle generation, or absorption? Based on the molecular cloud's central location relative to both the NTFs and the Galactic plane, on the observed increase in en- 


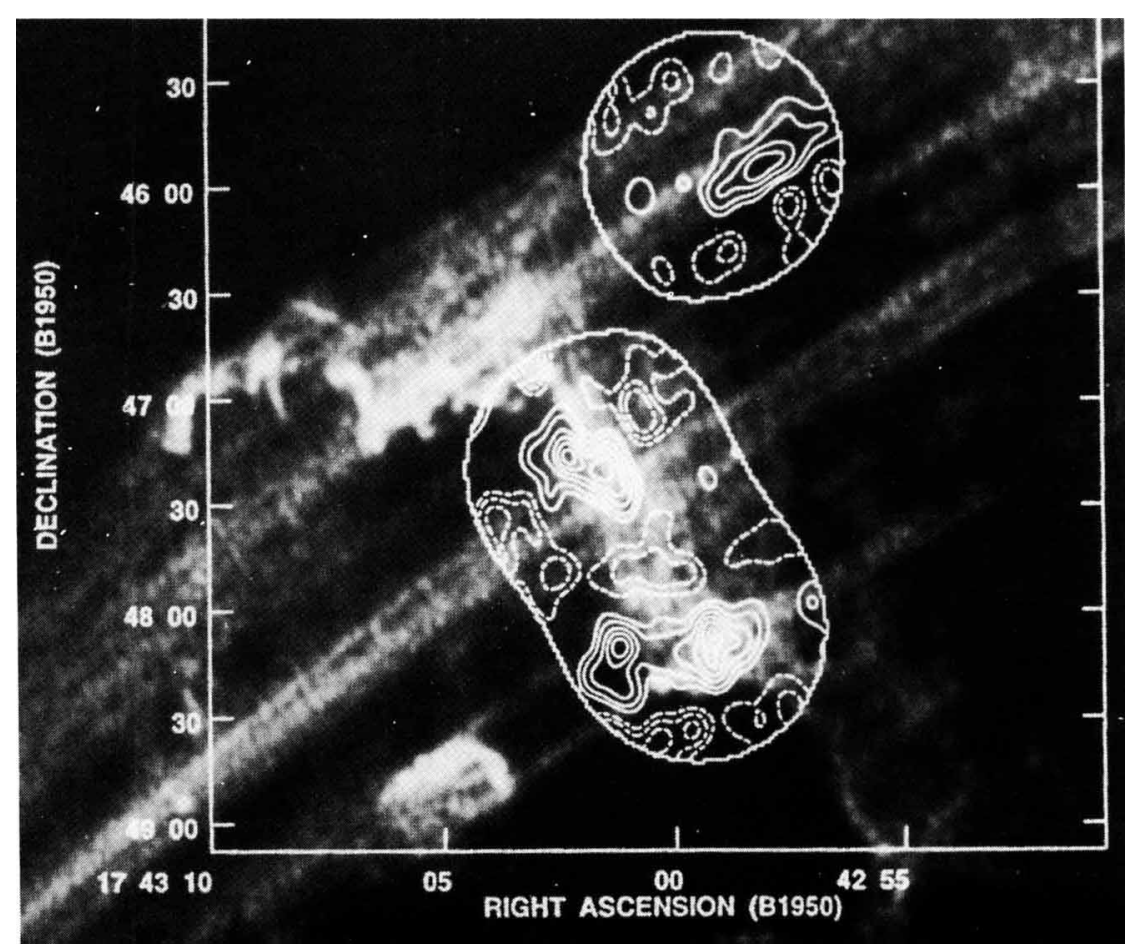

Figure 1. Superposition of the OVRO CS J=2-1 three-field mosaic of the $25 \mathrm{~km} \mathrm{~s}^{-1}$ cloud (contours) on a $5 \mathrm{GHz}$ radiograph of the G0.18-0.04 HII region and the nonthermal filaments in the Arc (from Serabyn and Morris 1994).

tropy of the Arc away from the Galactic plane (only amorphous plumes are seen at Galactic latitudes $>0.2^{\circ}$; Seiradakis et al. 1985 ; Tsuboi et al. 1986), and on the observed decrease in the Arc's spectral index away from the Galactic plane (Reich 1990), which argues for a more aged population of electrons far from the Galactic plane, it is possible to conclude that the molecular clumps are the generation sites of the relativistic electrons.

\section{Reconnection Scenario}

Because the Arc's synchrotron emitting filaments attest to the presence of a strong magnetic field, it is natural to look to the magnetic field for the particle acceleration mechanism. This then brings us back to the question raised earlier - as a partially ionized molecular cloud attempts to traverse a region of high magnetic field, how does it interact with the field? The problem of course is that the cloud's internal field is frozen in by the small ionized component present, and so as the cloud traverses the ambient field, 
both the internal and external fields must deform to enable the passage of the intruding cloud (unless the ionization level is very low). The external field ahead of the cloud is thus compressed by ram pressure, and then swept around and past individual dense clumps, while at the same time, the clumps' internal field lines are dragged through the ambient medium. In analogy to the case of a magnetized planet making its way through the solar wind, a contact discontinuity develops ahead of each advancing cloud clump, wherein the compressed external field is forced into contact with the clump's own internal field. (In this analogy, we must of course remember that an interstellar cloud is not a rigid body, so that the external magnetic pressure likely leads to cloud fragmentation and interpenetration of the field and the clumps, and also that such a cloud's internal magnetic field is not internally generated, as in the case of a magnetized planet, but is instead a relic of the cloud's own history as part of the ISM). Thus, a structure resembling a planetary magnetopause is set up ahead of each advancing clump, in which particle acceleration may occur. However, due to the fluid nature of molecular clouds, this 'magnetopause' is likely not a stable surface - indeed it likely distorts markedly as a result of spatial density and ionization variations, rotational and turbulent motions in individual clumps, and fragmentation resulting from magnetic pressure gradients.

In the boundary layer ahead of the advancing clumps, the external and internal fields, which are likely dissimilar both in magnitude and orientation, are forced into contact, leading to the "reconnection" of the two fields. Briefly (Parker 1979), the two fields diffuse through the partially ionized medium toward the boundary, and this diffusion of flux induces an electric field which rapidly accelerates the free charges present. A more formal way to describe the phenomenon is that the curl of the magnetic field across the boundary necessitates a current flow (and so an electric field) along the boundary. Of course shocks may aid in the acceleration process. Once out of the interaction zone, the accelerated particles attach themselves to the nearest large scale field lines, and spiral vertically out of the plane of the Galaxy. Given the high frequency spectral cutoff of the NTF emission (between 15 and $43 \mathrm{GHz}$; Sofue et al. 1992), Gev electron energies and roughly $10^{4} \mathrm{yr}$ radiative lifetimes are indicated, implying that the relativistic electrons can easily travel the length of the filaments during their lifetimes.

In this scenario, the observed nonthermal filaments result from the local injection of particles onto ambient field lines from 'hot spots' corresponding to individual molecular clump surfaces. As a result, there is no need to rely on special magnetic field configurations based on the existence of a high field flux tube at the location of the Arc (which would raise pressure balance problems). Instead, even with a uniform magnetic field structure, all that is required is a clumpy molecular medium, which is in fact observed. Since 
reconnection likely also occurs at a lower level in the more diffuse interclump medium, this scenario can also explain the more extended 'background' of nonthermal emission seen around the individual nonthermal filaments. Indeed, fewer collisions near the generation site may allow electrons from more diffuse gas to escape with higher energies, consistent with the higher cutoff frequency seen outside of the well-defined filaments (Sofue et al. 1992).

The details of the reconnection and acceleration processes are beyond the scope of this paper (and also occur on a scale too small to be directly observable at the distance of the Galactic center), but two general questions can be addressed. First, is the process energetically feasible - i.e., is the magnetic energy swept up by the advancing cloud sufficient to power the luminosity seen from the relativistic particles in the Arc? Second, since the process described is quite general, why is the Arc such a unique source?

To address the first question, a cloud coursing thru an ambient field encounters a magnetic energy flux of $10 B^{2} v \mathrm{~L}_{\odot} / \mathrm{pc}^{2}$, where $B$ is the external field in $\mathrm{mG}$, and $v$ is the cloud-field relative velocity in $\mathrm{km} \mathrm{s}^{-1}$. Allowing for a cloud cross-section of $10 \mathrm{pc}^{2}$, a field of $1 \mathrm{mG}$, and a relative velocity of $100 \mathrm{~km} \mathrm{~s}^{-1}$ (all of which are subject to large uncertainties), the $25 \mathrm{~km} \mathrm{~s}^{-1}$ cloud then encounters magnetic energy at a rate of $\sim 10^{4} \mathrm{~L}_{\odot}$. In contrast, the luminosity of the Arc is only of order $10^{2} \mathrm{~L}_{\odot}$, requiring an efficiency for magnetic to kinetic energy conversion of only $1 \%$, a level consistent with theoretical expectations for field reconnection (SM).

Concerning the second question, since the rate of accessing magnetic energy depends on the ambient magnetic energy density, as well as on the cloud-field relative velocity, clearly a large luminosity in relativistic particles can be produced only in the central high-field, high-turbulence region of our Galaxy. However, while this volume includes many molecular clouds, the instances of filamentation are relatively few, leading one to suspect that the picture is not yet complete. Indeed, a further variable is involved the ionization structure of the cloud. This enters in two opposing ways, as the reconnection rate is inversely proportional to the conductivity of the medium (and so is low if the ionized fraction is high), but free charges must be present to provide an ionized population susceptible to acceleration.

Presumably what occurs is then the following. If the ionization level is low, a projectile cloud or clump can easily be accomodated by the fields, although some very low level reconnection may occur. If however, a cloud has an ionized surface layer (presumably due to photoionization, but perhaps aided by Alfven's critical ionization effect), the cloud's projectile motion will be strongly opposed by the ambient field. With ionized surfaces which are oriented more or less along the direction of motion, the ionized layers can shear off of the bulk of the neutral medium, disrupting the cloud, 
but likely leading to little in the way of reconnection. This may in fact explain the elongated appearance of the nearby thermal "arched filaments" (Morris, this volume). On the other hand, if a cloud's leading surface were ionized, the results would be dramatic: the approaching fields would be pressed against each other, and prolific reconnection and particle acceleration would occur at the interface, as discussed above. Thus, by combining the need for a high magnetic field, a high cloud-field relative velocity, and also surface ionization in the leading cloud layers, the phenomenon seen as the Galactic Center Arc should indeed be rare.

\section{References}

Bally, J., Stark, A.A., Wilson, R.W., \& Henkel, C. 1988, Ap. J. 324, 223.

Güsten, R. 1989, in IAU Symp. 136, "The Center of the Galaxy", ed. M. Morris (Dordrecht: Kluwer), 89.

Lasenby, J., Lasenby, A.N., \& Yusef-Zadeh, F. 1989, Ap. J. 343, 177.

Morris, M. 1994, in "The Nuclei of Normal Galaxies: Lessons from the Galactic Center", eds. R. Genzel \& A. Harris (Dordrecht: Kluwer).

Parker, E.N. 1979, Cosmical Magnetic Fields (Oxford: Clarendon Press).

Reich, W., 1990 in IAU Symp. No. 140, "Galactic and Intergalactic Magnetic Fields", eds. R. Beck, P. Kronberg and R. Wielebinski (Dordrecht: Kluwer), 369.

Seiradakis, J.H., Lasenby, A.N., Yusef-Zadeh, F., Wielebinski, R., \& Klein, U. 1985, Nature 317, 697

Serabyn, E. \& Güsten, R. 1991, Astr. and Ap. 242, 376.

Serabyn, E. \& Morris, M. 1994, Ap.J. Lett. 424, L91.

Sofue, Y., Murata, Y. \& Reich, W. 1992, Publ. Ast. Soc. Japan, 44, 367.

Sofue, Y., Reich, W., Inoue, M., \& Seiradakis, J.H. 1987, Publ. Ast. Soc. Japan 39, 95.

Tsuboi, M., Inoue, M., Handa, T., Tabara, H., Kato, T., Sofue, Y., \& Kaifu, N. 1986, AJ, 92, 818.

Yusef-Zadeh, F. \& Morris, M. 1987, Astron. J. 94, 1178.

Yusef-Zadeh, F., Morris, M., \& Chance D. 1984, Nature 310, 557. 